\title{
Particle transport in tokamak plasmas, theory and experiment
}

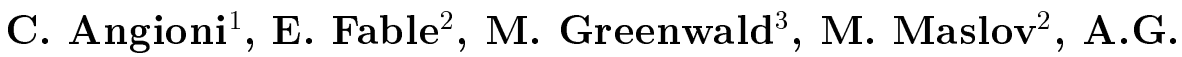 \\ Peeters $^{4}$, H. Takenaga ${ }^{5}$, H. Weisen ${ }^{2}$ \\ 1 Max-Planck Institut für Plasmaphysik, IPP-EURATOM Association, D-85748 \\ Garching bei München, Germany \\ 2 Centre de Recherches en Physique des Plasmas, Association \\ EURATOM-Confédération Suisse, EPFL, 1015 Lausanne, Switzerland \\ 3 Plasma Science and Fusion Center, Massachusetts Institute of Technology, \\ Cambridge, $M A, U S A$ \\ ${ }^{4}$ Centre for Fusion, Space and Astrophysics, University of Warwick, CV4 7AL, \\ Coventry UK \\ 5 Japan Atomic Energy Agency, 801-1 Mukouyama, Naka, Ibaraki 311-0193, Japan
}

\begin{abstract}
The physical processes producing electron particle transport in the core of tokamak plasmas are described. Starting from the gyrokinetic equation, a simple analytical derivation is used as guidance to illustrate the main mechanisms driving turbulent particle convection. A review of the experimental observations on particle transport in tokamaks is presented and the consistency with the theoretical predictions is discussed. An overall qualitative agreement, and in some cases even a specific quantitative agreement, emerges between complex theoretical predictions and equally complex experimental observations, exhibiting different dependences on plasma parameters under different regimes. By these results, the direct connection between macroscopic transport properties and the character of microscopic turbulence is pointed out, and an important confirmation of the paradigm of microinstabilities and turbulence as the main cause of transport in the core of tokamaks is obtained. Finally, the impact of these results on the prediction of the peaking of the electron density profile in a fusion reactor is illustrated.
\end{abstract}

\section{Introduction and motivations}

Research on particle transport is an essential element for understanding basic properties of turbulence in magnetically confined plasmas and plays a crucial role toward the achievement of practical fusion energy.

Since fusion power scales with the square of the density, obtaining high values of the central density in a reactor is of particular importance. Recent studies [1] show that the cost of electricity produced by a fusion power plant is proportional to $\beta_{N}^{-0.4}\left(n / n_{G}\right)^{-0.3}$, where $\beta_{N}=\beta_{T} /\left(I_{p} / a / B_{T}\right)$ is an appropriately normalized thermal plasma pressure, with $\beta_{T}=2 \mu_{0}<n T>/ B_{T}^{2}, B_{T}$ the toroidal magnetic field, $I_{p}$ the plasma current and $a$ the minor radius, while $n / n_{G}$ is the ratio of the plasma density to the density 
limit, observed in tokamaks and calculated by means of the Greenwald empirical scaling [2]. This relationship shows that efficient operation of a fusion reactor requires at least as much attention to achievement of high density as to reaching high temperature or high pressure. This point is underlined by recent conceptual studies for a future power plant [3], where densities are required to exceed the empirical limit by a factor of 1.2 to 1.5. Reaching such very high densities is only possible with a centrally peaked density profile, which allows the density at the plasma periphery to remain below the value of the density limit. It has been observed in tokamaks that plasmas with peaked density profiles could exceed the density limit by keeping the edge density below the Greenwald limit $[4,5,6,7,8]$.

Centrally peaked density profiles are regularly observed in tokamaks, however the degree of peaking can change significantly depending on the plasma regime. In most cases the particle source is only peripheral, which implies that the observed peaking requires the existence of a particle influx, namely a pinch, which balances the diffusive outward flux in the presence of a nonzero density gradient. In a tokamak, the conservation of the canonical angular momentum in combination with the presence of the induced toroidal electric field, leads to an inward flow of trapped particles [9]. While this neoclassical mechanism of pinch has been identified in some experiments $[10,11,5,12,13]$, in most conditions it cannot explain the observed peaking, since it is too small compared to the plasma diffusivity. The latter is measured to be of the order of the electron heat conductivity $[14,15,16,17,18,19,20]$ and therefore, like heat transport, largely above the collisional neoclassical predictions [21]. In most conditions, density peaking has an "anomalous", non-neoclassical, nature [22, 15, 23, 19], that is, it must be ascribed to turbulence. A recent confirmation comes from the observation of peaked density profiles in fully non-inductive discharges, that is tokamaks with no toroidal electric field [24, 25, 26, 27, 28].

Since in most cases the effects of the Ware pinch and of the particle source, produced by both wall neutrals and neutral beam injection (NBI), have a limited impact on the shape of the density profile, the density profile is mainly determined by the balance between the outward turbulent diffusion and the inward turbulent convection. In the last decade, experimental research has been intensively dedicated to the identification of the mechanisms which underly the density profile peaking and on its scaling with various local plasma parameters $[29,30,31,32,33,34,25,35,36,37,38,39,27,40,41$, $42,43,44,45,46,47,48,49,28,50,51,52,53,54]$. As a result of such a scientific effort, a rather complete picture of the behaviour of the density profiles in tokamaks emerges, which is drawn in Section 3.

Theoretical research was initially devoted to the identification of the main sources of the turbulent particle pinch. These were related first to the role of the electron temperature gradient $[55,56,57,58,59,60,61]$, within both fluid and kinetic models. Later, the role of magnetic field inhomogeneity and curvature was recognized, with a fluid theory [62, 63], delivering convection terms proportional to both the electron temperature gradient and the magnetic field curvature. A different methodology, in 
which turbulent fluxes are expressed in terms of adiabatic invariants, [64, 65, 66, 67, 68, 29], identified a pinch term proportional to the radial gradient of the tokamak safety factor. This pinch mechanism was confirmed by fluid simulations of interchange turbulence [69]. Subsequently, within the same adiabatic invariant approach, the introduction of a velocity dependence in the computation of the turbulent transport also led to a convection term proportional to the electron temperature gradient $[70,30]$.

More recently, it was clarified that the convective terms found by the previously applied, and complementary, fluid and adiabatic invariant approaches are actually equivalent $[71,72]$ and that fluid and kinetic models directly include both the electron temperature gradient term and the magnetic curvature term. A comprehensive theory in which thermodynamical forces and conjugated fluxes are rigorously defined in the expression of the entropy production rate in both fluid and kinetic regimes was subsequently developed [73]. In recent years, research has been devoted to the identification of the main dependences of the convective terms as a function of experimentally relevant parameters, applying both fluid [62, 63, 74, 75, 71] and kinetic [76, 77, 78, 79, 80, 81, 82] models and related codes. By these works $[35,36,37,12,38,83,84,85,42,86,87,88,89,90,54,91]$, important progress has been made in the theoretical description and understanding of the mechanisms producing particle transport, including the role of linearly stable modes [92], and in the interpretation and prediction of the complex and sometimes apparently controversial experimental observations.

These studies have revealed that the dependences of the turbulent pinch on local plasma parameters can change significantly, and even reverse, as a function of the type of instability and turbulent regime. An equally complex experimental phenomenology corresponds to the theoretical complexity. A unified and rather complete picture emerges today, which is the subject of this paper. Most of the experimentally observed dependences of density peaking can be explained in terms of theoretically predicted particle convection mechanisms within a single theory. This provides one of the most robust validations of the paradigm of microinstabilities and turbulence as the main cause of transport in the core of tokamaks, and indicates that the behaviour of the density profile can be interpreted as a macroscopic fingerprint of the type of turbulence present in the plasma.

In the next section the physical mechanisms leading to particle transport in a tokamak are reviewed from a theoretical standpoint, by means of a simple analytical derivation from the linearized gyrokinetic equation. In Section 3, the experimental dependences observed in different tokamaks and operational regimes are presented in a unified fashion, and compared to the theoretical predictions. In Section 4, concluding remarks and the implications on the extrapolation to the density profile of a plasma in a fusion reactor are briefly presented. 


\section{Theory}

Collisions are the basic (classical) mechanism which transports particles in a plasma. However, even including the enhancement of the diffusivity provided by the toroidal geometry [21], namely the neoclassical transport, the neoclassical diffusivity is approximatively $2 / 3$ of the neoclassical electron heat conductivity, and, like the latter, is experimentally negligible in all observed conditions. Neoclassical theory predicts the existence of non-diagonal contributions to the particle transport, that is particle fluxes proportional to both the electron and the ion temperature gradients, which are of the order of the diagonal coefficient (e.g. [93]), and an additional term, proportional to the induced toroidal electric field in the tokamak, the Ware pinch [9]. The latter is the only neoclassical mechanism of particle transport which was observed experimentally in tokamaks $[10,11,5,12,13]$, and which can be nonnegligible in some conditions. However, its effect becomes negligible at the high temperatures required in a fusion reactor.

The overall agreement between theoretical predictions and experimental observations, which will be reported in this paper, strongly suggests that the main source of particle transport in the core of a tokamak is provided by turbulence driven by microinstabilities at the ion Larmor radius scale, namely the ion temperature gradient (ITG) mode [94, 95] and the trapped electron mode (TEM) [96, 62]. The most appropriate framework to describe these instabilities is the gyrokinetic theory [97, 98, 99].

Passing electrons are very close to the adiabatic response, due to their fast motion along the field lines, unless a source of non-adiabaticity is introduced. A non-adiabatic electron response can be caused by high collisionality or electromagnetic effects, which are experimentally relevant in the plasma edge [100] or by very low magnetic shear, which tends to be relevant in the centre of the plasma column. For usual plasma parameters of fusion devices, the electron particle flux in the confinement region is mainly produced by trapped electrons.

Starting from the linearized electrostatic version of the gyrokinetic equation and by means of a simple formal analytical derivation, we identify the main mechanisms of particle transport produced by ITG and TEM relevant for the core of a tokamak. Analogous treatments can be found in [72], where the drift-kinetic equation was used, and in [89, 101]. The latter reference includes also collisional effects, and is suggested for a more complete and detailed derivation. The non-adiabatic part $g_{k}$ of the perturbed electron distribution function $f_{k}=g_{k}+F_{M} J_{0} \hat{\phi}$, for a single toroidal mode number $n=k_{y} r / q$, with $k_{y}$ the poloidal wave number, $r$ the local minor radius of the plasma, and $q$ the local safety factor, is decomposed in Fourier harmonics $g_{k}=\hat{g}_{k} \exp (-i \omega t+i \mathbf{k} \cdot \mathbf{x})$. In the simple $s-\alpha$ circular shifted geometry [102], described by a limited set of local parameters, the inverse aspect ratio $\epsilon=r / R$, the safety factor $q$, the magnetic shear $s=r d q / d r / q$, and $\alpha=-q^{2} R 8 \pi d p / d r / B^{2}$, with $p$ the total plasma pressure, the 
equation reads,

$$
\left(\omega-\omega_{G k}+i \nu_{e i}\right) \hat{g}_{k}=\left\{\omega_{D k}\left[\frac{R}{L_{n}}+\left(\frac{E}{T_{e}}-\frac{3}{2}\right) \frac{R}{L_{T e}}\right]-\omega\right\} F_{M} J_{0}\left(k_{\perp} \rho_{s}\right) \hat{\phi}_{k},
$$

where a simple Krook collision operator has been included, to describe electron- ion collisions, with a collision frequency $\nu_{e i}$. On the left hand side, the motion of the gyrocenters is described by the total frequency $\omega_{G k}=k_{\|} v_{\|}+\omega_{d k}$, where the derivative along the field line has been formally replaced by a parallel wave number $k_{\|}$. The perpendicular drift frequency arising from the $\nabla B$ and curvature drifts is $\omega_{d k}=$ $\omega_{D k}\left[\left(v_{\|}^{2}+v_{\perp}^{2} / 2\right) / v_{\text {the }}^{2}\right][\cos \theta+(s \theta-\alpha \sin \theta) \sin \theta]$, with $\omega_{D k}=k_{y} \rho_{s} c_{s} / R$ and $\theta$ the extended ballooning angle. At the right hand side, the normalized logarithmic gradients of the equilibrium density and temperature $R / L_{n}=-R d n / d r / n$ and $R / L_{T e}=-R d T_{e} / d r / T_{e}$ stem from the radial derivative of the equilibrium Maxwellian distribution $F_{M} . R$ is the major radius at the center of the magnetic flux surface, $E$ the kinetic energy of the particle, $c_{s}=\sqrt{T e / m_{i}}$ the sound speed, $\rho_{s}=c_{s} / \Omega_{c i}$ the ion Larmor radius computed with the sound speed, with $\Omega_{c i}$ the ion cyclotron frequency. Finally, the fluctuating electrostatic potential has been normalized as usual $\hat{\phi}_{k}=e \phi_{k} / T_{e}$.

The quasi-linear particle flux $\Gamma_{Q L}$ produced by the fluctuating $E \times B$ drift $\tilde{v}_{E \times B}$ is $\left\langle\tilde{n}_{e} \widetilde{v}_{E \times B}\right\rangle$, where the brackets express flux surface average.

By formally computing the linear phase shift between density and electrostatic potential fluctuations from Eq. (1), we obtain the following expression

$$
\Gamma_{Q L}=\sum_{k} \frac{k_{y} c_{s}^{2}}{\Omega_{c i}}\left\langle\int d^{3} v F_{M} \frac{\left(\hat{\gamma}_{k}+\hat{\nu}_{k}\right)\left[R / L_{n}+\left(E / T_{e}-3 / 2\right) R / L_{T e}\right]-\left(\hat{\gamma}_{k} \hat{\omega}_{G k}-\hat{\omega}_{r k} \hat{\nu}_{k}\right)}{\left(\hat{\omega}_{r k}+\hat{\omega}_{G k}\right)^{2}+\left(\hat{\gamma}_{k}+\hat{\nu}_{k}\right)^{2}} J_{0}\left(k_{\perp} \rho_{s}\right)^{2}\left|\hat{\phi}_{k}\right|^{2}\right\rangle,
$$

where $\gamma_{k}$ and $\omega_{r k}$ are the growth rate and real frequency of the unstable linear mode and all frequencies have been normalized to the fluid perpendicular drift frequency $\omega_{D k}=k_{y} \rho_{s} c_{s} / R$. The sign convention is that a positive value for the real frequency $\hat{\omega}_{r k}$ identifies a mode propagating in the ion diamagnetic direction, that is, ITG. The quasilinear flux is computed by considering the complex linear eigenfrequency $\omega_{r k}+i \gamma_{k}$, as is customary in fluid transport models $[62,63,75]$. It can be shown $[89,103]$ that this is equivalent to assume a turbulent frequency spectrum given by a Lorentzian distribution, whose width is given by the linear growth rate.

Eq. (2) shows that the quasi-linear particle flux has the following form made of three contributions,

$$
\frac{R \Gamma_{Q L}}{n_{e}}=D \frac{R}{L_{n}}+D_{T} \frac{R}{L_{T e}}+R V_{p}
$$

The first is diagonal diffusion, proportional to the logarithmic density gradient. The second is proportional to the logarithmic temperature gradient and plays the role of thermo-diffusion. The third is not proportional to any gradient of any kinetic profile, and is therefore a pure convective term. Obviously, the transport coefficients $D, D_{T}$ and the convective velocity $V_{p}$ are functions of the logarithmic gradients $R / L_{n}$ and $R / L_{T e}$, since a change of the gradients affects the instability, namely changes the eigenfrequencies and the phase relations between density and potential fluctuations. 
Therefore Eq. (3) provides a valid physical decomposition, but cannot be interpreted as a linear relationship.

Eq. (2) shows that the (diagonal) diffusion coefficient is positive, at all energies, namely it produces an outward directed flux for typical centrally peaked density profiles. The diagonal diffusion is produced by particle advection caused by the fluctuating $E \times B$ drift. The main instability leading to strong outward diffusion of particles is the density gradient driven TEM [94, 62], which is linearly unstable above a threshold in the normalized logarithmic density gradient $R / L_{n}$, and which produces a particle flux strongly increasing with increasing $R / L_{n}$. In experiments, such a mode can be expected to be stable or only marginally unstable, since otherwise the turbulent particle flux would exceed the volume integral of the particle source in the particle balance equation. This mode has been found of experimental relevance in conditions of high collisionality in Alcator C-Mod, where its destabilization was found to balance the inward flux produced by the neoclassical Ware pinch [12]. In that work, nonlinear simulations close to the linear threshold also revealed a nonlinear upshift of the critical logarithmic density gradient, similar to that observed for $R / L_{T i}$ in the ITG mode [104]. The effective nonlinear threshold for the onset of significant TEM particle flux increases strongly with collisionality [105], much more so than in the ITG case, while the linear threshold increases more weakly [86]. The nonlinear upshift can double the critical density gradient for C-Mod relevant collisionalities [105]. This can can lead to mechanisms of increasing density peaking with increasing collisionality in high collisionality plasmas.

In contrast to diagonal diffusion, which is always directed outwards, Eq. shows that both off-diagonal contributions, namely the thermodiffusion and the pure convection, can be directed inward or outward depending on the plasma parameters.

\subsection{Thermodiffusion}

The thermodiffusion coefficient includes the energy kernel $E / T_{e}-3 / 2$ derived from the Maxwellian in the presence of a background temperature gradient. We now consider the collisionless limit. Obviously, if the frequency $\hat{\omega}_{G k}$ in the denominator were energy independent, this term would be identically zero, since the integration in the velocity space over the Maxwellian would produce a perfect balance between slow particles moving inward and fast particles moving outward. However, in a magnetized plasma, the drift frequency does depend on energy and the term must be included. At this point it is useful and practical to distinguish between the toroidal resonance, given by the perpendicular drift frequency $\omega_{d}$ and the slab resonance given by $k_{\|} v_{\|}$. Both resonances lead to a finite value of $D_{T}$.

We first consider the toroidal resonance, which involves trapped electron dynamics and is, in general, relevant for core transport phenomena. Since the vertical drift is proportional to the particle energy and depends on whether the unstable mode is propagating in the ion or in the electron drift direction, (that is with a positive or negative sign of $\hat{\omega}_{r}$ in Eq. (2)), the perturbed distribution function implies an increase 
of particles in the low or in the high energy range with respect to the Maxwellian, leading to a lack of balance along the energy axis, and therefore to a net flux. In the case of ITG modes, with positive value of $\hat{\omega}_{r}$, this amplifies the inward contribution of slow particles, with consequent net inward flux. This increases in the inward direction for values of $\hat{\omega}_{r}$ which approach or even cross zero but remain weakly negative.

Instead, for values of $\hat{\omega}_{r}$ which are large and negative, that is in conditions of strong TEM instabilities, the outward contribution from large energies becomes dominant, and induces a net flux directed outwards. This term, due to the toroidal resonance in the collisionless limit, was first derived from a fluid model $[62,63]$. There, it stems naturally from the coupling between density fluctuations and temperature fluctuations produced by the energy dependence of the vertical drift. In such a model it is found that the condition for the thermodiffusive flux to be directed outwards is given by $\hat{\omega}_{r}<-10 / 3$. Later, the same term was identified with a complementary approach, based on adiabatic invariants [70,30]. In collisional regimes, this term was actually identified earlier [58, 60, 61], from kinetic equations. It was first recognized that it was directed outwards in the high collisionality regime for ITG modes [58], and then observed that it can reverse direction and become directed inwards in low collisionality regimes $[60,61]$. Recent quasi-linear gyrokinetic calculations $[38,101]$ show that it is directed inwards in the ITG domain over a wide range of experimental collisionalities, although its strength decreases at very large collisionalities. The interesting feature, that it can reverse direction from inward to outwards when strong TEM modes are excited, was noted only in recent works [36, 71, 38, 42], in relation with the experimental observations of density flattening with central electron heating (see Sec. 3). The reversal from inward to outwards has been also confirmed by nonlinear fluid simulations [71].

The slab resonance $k_{\|} v_{\|}$, relevant for the passing electron response, also leads to a net thermodiffusive flux. If a high collisionality regime is assumed, as described by the Braginskii equations [106], then the coupling mechanism between density fluctuations and temperature fluctuations is provided by the non-adiabaticity of the passing electron response produced by parallel thermal forces, and leads to an inward flux of passing electrons proportional to the electron temperature gradient. This was actually the first effect to be pointed out in the literature leading to an anomalous pinch [55]. This effect was confirmed with nonlinear fluid simulations [57]. It is interesting to note that in the collisionless limit a net thermodiffusive flux is also produced by the slab resonance, carried by the very slow passing electrons which fulfil the resonance $v_{\|}=\omega_{r} / k_{\|}[70,107]$. Starting from Eq. (2), such a flux can be computed analytically, in the limit where a delta function in $v_{\|}$is assumed for the resonating particles and the asymptotic value of $D_{T} / D=-1 / 2$ is obtained [101], which agrees with the value found in [70] by means of a Hamiltonian approach. 


\subsection{Pure convection}

We turn to the pure convective term $V_{p}$, by first discussing the more relevant toroidal resonance, which is important for the trapped electron response. In the collisionless limit, convection is proportional to the vertical drift frequency $\omega_{d k}$. Hence, it is a purely geometrical effect arising from the inhomegeneity and curvature of the magnetic field, and for this reason it is often dubbed curvature pinch. In the limit of an instability strongly ballooning at the low field side and represented by a delta function, it can be computed analytically as $V_{p}=-2 D / R[62,63]$. More generally, it is inversely proportional to the major radius $R$ of the flux surface, and it has to be computed considering the actual mode structure along the field line. It depends on parameters describing the magnetic equilibrium geometry (as indicated by the dependence on shear and $\alpha$ of $\omega_{d}$ reported above in the $s-\alpha$ model). For usual monotonic safety factor profiles, it is directed inwards, and increases in size with increasing magnetic shear. It can reverse direction in the case of unstable modes with negative shear and/or a strong shift of the magnetic axis (i.e. large values of $\alpha$ in the $s-\alpha$ model). This term was originally identified within a fluid description $[62,63]$ and later by an independent approach based on the description of turbulent fluxes in terms of adiabatic invariants, and in particular with models of turbulent equipartition [64, 65, 66, 67, 68, 69, 29, 70, 30]. It is produced by the perpendicular compression of the $E \times B$ drift [62,63, 71], and can be properly expressed in terms of canonical profiles within an appropriate definition of thermodynamic forces, and related fluxes, in the expression of the entropy production rate [73]. This term is included naturally in the gyrokinetic equation and therefore it is present in any gyrokinetic code describing tokamak core instabilities and turbulence, as well as in the fluid models usually applied for transport modelling [62, 75, 108], although with different levels of approximation.

It is of interest, and great experimental relevance, to look at the effect of collisions on this term. As we can see from Eq. (2), a non-zero collision frequency implies an additional term, which, within our very simple analytical description, is proportional to both the collision frequency and to the real frequency of the unstable mode. For ITG modes, this term is directed outwards, and, at large collisionalities, it reduces or reverses the inward contribution of the collisionless curvature pinch term. This effect was first identified by means of transport simulations of AUG plasmas [35, 36] with collisional and collisionless fluid models [75, 62]. The effect was confirmed in both linear [86] and nonlinear [85, 91] gyrokinetic simulations, and recently found to agree quantitatively with the experimentally observed dependences [54]. In the case of instabilities with negative drift frequency, that is those propagating in the electron drift direction, the collisional term is directed inwards [91, 101]. We note that an increase of collisionality always implies an increase of the real mode frequency, which for ITG modes amplifies the collisionality effect, while for TEM modes provides a compensation. Hence, in the latter case, the effect of collisionality is predicted to be rather weak.

It is also worth noting that collisions strongly impact the dependence of the 
curvature pinch on magnetic shear [101]. In particular, it is found that the value of $R / L_{n}$ which fulfils the condition of zero particle flux, increases with increasing shear in the collisionless limit or in the case of dominant TEM, but becomes almost independent of shear or even reverses the dependence when a dominant ITG is considered in the presence of collisions. This result has some important implications in the interpretation of the experimental observations, which are presented in the next Section.

We now consider the slab resonance relevant for the passing electron response. In the collisionless limit, a passing electron convection is present, arising from parallel compression of parallel velocity fluctuations. Analogously to the collisionless thermodiffusion of passing electrons, it can be computed analytically by treating the slab resonance with a delta function. For a single mode number $k_{y}$, it is found that $V_{p k}=\left(T_{e} / T_{i}\right) \hat{\omega}_{r k} D_{k}$ [101], and therefore it is directed inward for modes propagating in the electron drift direction, and outward for ITG modes. The same process was pointed out recently for impurity transport [109], where the dependence on the mode frequency is reversed, due to the opposite sign of the particle charge. An analogous convective transport mechanism is produced by electromagnetic induction, leading to a convection of passing electrons, which is directed inward for modes propagating in the electron diamagnetic direction, and outward for ITG modes [110]. The latter effect has been also observed in nonlinear gyrokinetic simulations [111]. Inward convection of passing electrons in the electrostatic limit has been also found in gyrokinetic simulations [87]. Of course the experimental relevance of pinch mechanisms carried by passing electrons has to be considered with care. Any pinch produced by passing electrons is of the same order of magnitude as the diffusivity of the passing electrons, which is roughly a square root of the ion to electron mass ratio smaller than the diffusivity of the trapped electrons, unless a cause of strong non-adiabaticity of passing electrons is present. Passing particle effects can therefore have experimental relevance at the plasma edge or, possibly, very close to the magnetic axis [112], but are expected to play only a limited role in the confinement region.

The existence of a particle pinch has been confirmed in both fluid [71] and gyrokinetic [84, 85, 88, 91] simulations of tokamak core plasma turbulence. The experimentally relevant condition of particle flux close to null is met by a balance between outward and inward contributions occurring at small and large toroidal mode numbers respectively [91]. The consistency between the theoretical predictions and the experimental observations encompassing not only particle transport, but concurrently also the electron and ion heat and momentum transport channels, is considered as an increasingly stringent test for the theory, on the road towards a more complete understanding of transport in tokamaks. In combination to gradient driven nonlinear gyrokinetic simulations, more challenging flux driven nonlinear gyrokinetic simulations can be regarded as a more realistic approach in this validation effort. In additon, nonlinear simulations give crucial constraints which are progressively taken into account in the development of present quasi-linear models [108, 89, 103, 113], where quasi-linear calculations are still required not only for transport modelling but also for dedicated 
quantitative comparisons over large experimental datasets [54]. In this framework, it is also important to note that the role of linearly stable modes has been pointed out, and found to lead to a pinch in the presence of TEM turbulence [92].

\section{Experiment}

Recent experimental studies have demonstrated the existence of a turbulent particle pinch through the observation of a peaked density profile in fully non-inductive discharges, [24, 25, 26, 27, 28], confirming previous conclusions based on plasmas in inductive scenarios $[22,23,19]$. In discharges without auxiliary NBI heating, the impact of the particle source produced by wall neutrals on the observed density peaking has been investigated and found to be negligible [24,44]. In the presence of NBI, the role of the particle source depends critically on the assumption made on the size of the diagonal diffusion coefficient $[37,40,44]$. For diffusion coefficients which are of the same order of magnitude of the power balance heat conductivity, as suggested by theory and as found in transient transport $[14,15,20,114]$ or trace Tritium [115] experiments, the effect of the beam particle source can be estimated to be no larger than $20 \%$. This is consistent with the comparison between plasmas with on-axis and off-axis NBI heating or with radiofrequency heating [34,45], as well as with the results delivered by statistical analyses over large databases $[46,51]$.

Analogously, in an inductive scenario, any stationary central peaking of the density profile can be explained by the Ware pinch provided that a sufficiently small diffusivity is assumed. Again, this assumption should not be inconsistent with measurements of the electron diffusivity and convection performed in transient transport experiments. The time evolution of the density peaking in transient phases can be used to identify the dominant role of the Ware pinch in providing the peaking, as observed in phases of slow density rise, connected with an increase of central peaking, in very high density plasmas (e.g. [5]). As already mentioned, an interesting case is the strong central peaking of the density profiles obtained with off-axis radio frequency heating in Alcator $\mathrm{C}-$ mod, where the inward flux produced by the Ware pinch is balanced by the outward flux produced by the destabilisation of a density gradient driven TEM, leading to steady conditions [12]. In most conditions, Ware pinch and particle source play a minor role in determining the peaking of the density profile, and cannot be expected to lead to any significant peaking in a high temperature and high density plasma of a fusion reactor.

Transient experiments have provided clear evidence that electron particle transport and density profiles cannot be explained as consequences of neoclassical and particle source effects in most cases $[14,15,16,17,18,20]$. The critical observation is that [20] diffusion and convection are in general comparable and of the same order of the heat conductivities, and scale in similar ways. Therefore, large convection velocities are measured in combination with large diffusivities. Contrary to neoclassical predictions, measured diffusion coefficients decrease with increasing density and in some cases increase with increasing temperature, and decrease with increasing safety factor. Finally, 
diffusion coefficients increase with increasing minor radius, from the centre to the periphery of the plasma. All of these experimental findings are not inconsistent with the predictions of turbulent transport. In particular, the dependences on density and temperature could be consistent with the theoretically predicted dependence of turbulent diffusion and convection on the plasma collisionality [36]. In addition, the increase of diffusion with minor radius can be be explained, in combination with other effects, by an important role for trapped electrons, whose fraction increases with the minor radius. All of these results are also consistent with the observation that the Ware pinch becomes particularly significant in the centre of high collisionality plasmas.

In recent years, important progress in the characterization of particle transport produced by turbulence has been obtained by concurrently investigating parameter dependences in large databases and performing dedicated experiments over a limited number of discharges. From all these studies, robust and coherent experimental evidences of a set of parametric dependences of density peaking can be identified.

Observations of L-mode plasmas in TFTR [66], DIII-D [29, 30], TCV [32] and JET [27] provide consistent evidence of a correlation between the peaking of the electron density profile and the peaking of the current density profile. The same dependence has been documented in specific experiments in Tore Supra (TS) [39] and FTU [28]. Interestingly, a positive correlation between local shear and local logarithmic density gradient $R / L_{n}$ was found in TS concurrently with a negative correlation between local logarithmic electron temperature gradient $R / L_{T e}$ and $R / L_{n}$, namely outward thermodiffusion [39]. On the contrary, when a positive correlation between $R / L_{n}$ and $R / L_{T e}$ is observed, namely inward thermodiffusion, the dependence on shear is found to be reversed and weak. Gyrokinetic microinstability analyses of those discharges found that in the latter condition the dominant instability is an ITG, while in the first case, where outward thermodiffusion is observed, the dominant unstable mode is a TEM.

The experimental relevance of outward thermodiffusion produced by TEM instabilities was first pointed out in relation with the outward convection of particles as a consequence of central electron heating in AUG plasmas [38], a phenomenon often dubbed density pump-out in the literature, and observed in several devices $[116,117,118,31,25,45,119,120]$. In agreement with the results provided by the analysis of TS discharges, outward thermodiffusion was found to take place in discharges where related linear gyrokinetic calculations identified a dominant TEM instability, while in conditions of dominant ITG, no effect of density pump-out was observed. This phenomenology is different from the density flattening observed in response to wave heating in high density plasmas, which can be produced by an increase of diffusivity and, in some cases, a reduction of the Ware pinch [5, 34, 36, 38]. The same conclusions with respect to the direction of thermodiffusion and the dominant instability at play are obtained in a study performed on FTU [28], similar to that carried out in TS [24]. The dominant role of inward thermodiffusion at the transition between ITG and TEM has been identified in TCV internal transport barriers, in fully non-inductive scenarios $[48,90]$, where unstable modes with real frequencies close to zero maximizes inward 


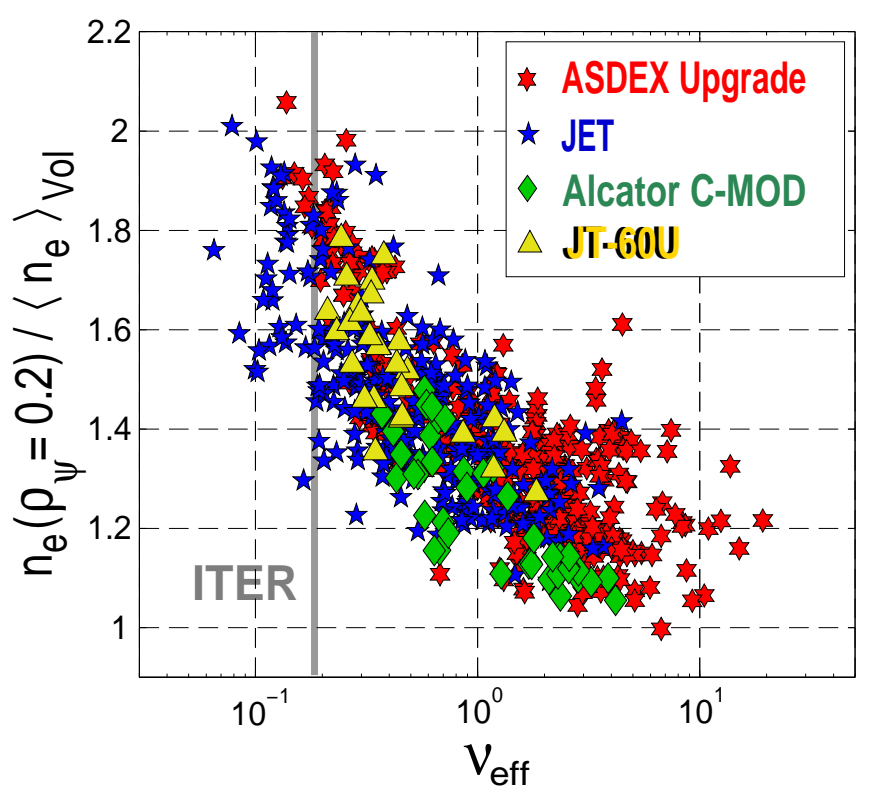

Figure 1. (Color online) Density peaking as a function the effective collision frequency from four devices, AUG [35], JET [41], C-Mod [52] and JT-60U [53].

thermodiffusion, as illustrated in Section 2 [90].

We shall see that the reversal of the direction of thermodiffusion in response to a change of dominant instability [38, 39, 42] is paradigmatic of a rather large set of behaviours of density peaking with respect to various plasma parameters. Indeed, the additional observation [39] of a strong reduction, or even reversal, of the dependence of $R / L_{n}$ on the magnetic shear in conditions where the thermodiffusion is measured to be inward might not be incidental. Likely, it is produced by the same physical process which is responsible of the observation of a weak or even negligible correlation between density peaking and current density profile peaking in $\mathrm{H}$-mode plasmas [41, 46, 51], contrasting with the strong correlation between density peaking and current density profile peaking observed in $\mathrm{L}$-mode plasmas [30, 32, 27, 39, 28]. These observations are consistent with the theoretical result, reported in Section 2, and obtained in linear gyrokinetic calculations including collisions [101], that, at the null of the flux, an increase of $R / L_{n}$ with increasing shear is predicted to occur in the presence of a dominant TEM instability, while it is not predicted to occur in the presence of a dominant ITG instability. The conclusion that the different behaviour is a consequence of different instabilities is supported by the observation that for datasets where a clear correlation between density peaking and current density profile peaking is identified, the majority of observations have $T_{e} / T_{i} \gtrsim 2$ and $R / L_{T e} \gtrsim R / L_{T i}[32,27]$. These are typical of a dominant TEM instability. In contrast, datasets without a clear correlation between density peaking and the current density profile peaking, are typically from plasmas in $\mathrm{H}$-mode with $T_{e} \simeq T_{i}$ and $R / L_{T e} \simeq R / L_{T i}[41,46,51]$, where the ITG instability is predicted to be the most unstable mode. The excitation of different instabilities therefore impacts the behaviour of density peaking not only with respect to thermodiffusion, but 
also with respect to its dependence on the peaking of the current density profile.

In the previous section we noted that the dependence of density peaking as a function of collisionality can be expected to behave differently in domains of parameters of dominant ITG compared to those of dominant TEM. Experimental observations are largely consistent also with these theoretical predictions. A weak or negligible dependence of density peaking on collisionality has been reported in L-modes in TCV [32], JET [46] and in $\mathrm{H}$-modes in TCV [121, 122]. A moderate increase of density peaking with increasing collisionality has been documented in FTU in fully noninductive plasmas [28]. The largest number of observations in these datasets feature $T_{e} / T_{i} \gtrsim 2$ and $R / L_{T e} \gtrsim R / L_{T i}$, and therefore can be predicted to be in a domain of dominant TEM instability. As illustrated in Section 2, in the case of TEM only a very weak dependence of increasing density peaking with increasing collisionality is expected. In particular, it is speculated here that the observation of an increase of density peaking with increasing collisionality in fully non-inductive plasmas with $T_{e} / T_{i} \approx 3$ [28] can be understood from the theoretical standpoint as a consequence of the effect of collisionality in the presence of dominant TEM instabilities. A decrease of collisionality can be also observed to correlate with a decrease of density peaking as a consequence of central electron heating in L-mode low density plasmas [119, 120]. However, in these conditions, the identification of the role of collisionality alone is hampered by the concurrent increase of the electron to ion temperature ratio [42], leading to the process of density pump-out discussed above.

Unlike the case of TEM instabilities, for plasmas with dominant ITG modes an increase of collisionality is predicted to strongly reduce the inward convection and to lead to a flattening of the density profile. This behaviour is indeed observed in tokamaks, in typical $\mathrm{H}$-mode plasma conditions, with $T_{e} \approx T_{i}$. In Fig. 1 the density peaking $n_{e}\left(\rho_{\psi}=0.2\right) /\left\langle n_{e}\right\rangle$, measured in $\mathrm{H}$-mode plasmas from four different devices, AUG [35, 36], JET [41, 46], Alcator $\mathrm{C}-\bmod [52]$, and JT-60U [53], is plotted as a function of the effective collisionality $\nu_{\text {eff }}=0.1 Z_{\text {eff }}\left\langle n_{e}\right\rangle R /\left\langle T_{e}\right\rangle^{2}[35,51]$, where the symbol \langle\rangle stands for volume average, $T_{e}$ is in $\mathrm{keV}, n_{e}$ in $10^{19} \mathrm{~m}^{-3}$, and $R$ in $\mathrm{m}$. This definition of normalized collision frequency provides an estimate of the ratio between the collision frequency and the drift frequency at the relevant scales for ITG and TEM transport [35, 36]. Statistical analyses of the experimental databases have shown that collisionality is indeed the parameter which has the largest bivariate correlation with density peaking as well as the largest statistical significance and statistical relevance in regressions $[46,51,52]$. An independent study, performed on experimental results from JET in the most recent campaigns, confirmed the previous results [54]. In particular, the combination of observations from AUG and JET with those from Alcator C-Mod allowed us to strongly reduce the covariance between collisionality $\nu_{\text {eff }}$ and the fraction to the density limit $n_{e} / n_{G}$, leading to the identification of $\nu_{\text {eff }}$ as the most appropriate parameter for extrapolations to ITER [52]. In addition, a quantitative agreeement has been found in a detailed comparison between the density peaking dependences observed in the JET database and the predictions of quasi-linear gyrokinetic calculations [54]. 
The latter result strongly supports the interpretation of the experimentally observed dependence as a macroscopic consequence of the effect of collisions on the inward curvature pinch carried by trapped electrons, in ITG turbulence.

\section{Conclusions}

Turbulence in fusion plasmas produces off-diagonal contributions to the particle flux, whose direction, outward or inward, depends on the type of instability, specifically on the direction of propagation of turbulent eddies. The overall agreement between theoretical predictions and experimental observations presented in this paper indicates that density peaking, and its dependences on plasma parameters, can be interpreted as a macroscopic fingerprint of the turbulent state present in the plasma, particularly with respect to the role of ITG and TEM instabilities. This result has already received some support from observation of fluctuations [123], though more direct confirmation awaits additional measurements, particularly the challenging measurement of mode propagation in the plasma frame. The current level of physical understanding may be deep and wellfounded enough to rely on for the prediction of density profile peaking in a fusion reactor.

A fusion reactor will operate with densities close to the density limit simultaneous with collisionality as low as the lowest values realized in present large tokamaks. This condition is a function of its very small $\rho^{*}=\rho_{s} / a$ and cannot be matched on current devices. Nevertheless, in present devices, when a peaked density profile is obtained at very high densities, the density limit can be exceeded in the plasma center. Such a peaking is usually generated by the Ware pinch at high collisionalities. This effect cannot be expected to play any significant role in a reactor. However, at the low collisionality of a fusion reactor, the particle pinch produced by turbulence can be expected to be at play, in the same way as it is at play in low collisionality $\mathrm{H}$-modes in present devices. Specifically, the ITER standard scenario can be expected to have a density profile with a density peaking $n_{e}\left(\rho_{\psi}=0.2\right) /\left\langle n_{e}\right\rangle=1.4-1.6$, as predicted consistently by empirical scalings $[46,50,51,52]$ as well as by theory-based transport simulations [124], where it is found to correspond to a local value of the logarithmic density gradient at mid-radius of $R / L_{n}=2-2.5$. The same value of local gradient is predicted by nonlinear flux-tube gyrokinetic simulations [91].

With such a peaking of the density profile, extrapolations performed on the basis of observations in JET H-modes [46] indicate an increase of the fusion output of the ITER standard scenario by approximately $30 \%$ with respect to a flat density profile, in agreement with the results of transport modelling [124]. Of course, more precise predictions depend on whether the extrapolation is made at fixed total particle content or at fixed edge density or at fixed plasma pressure [46]. Finally, it has to be stressed that the peaking of the density profile observed in low collisionality $\mathrm{H}$-mode plasmas in present devices is not observed to cause a concurrent flattening of the temperature profiles $[46,125,51,53,54]$. Operational scenarios for ITER should take account of the 
predicted peaked density profile as well as the concomitant change in pressure profile. Acknowledgments

One of the Authors (C.A.) is grateful to X. Garbet, C. Bourdelle, D. Ernst, G.T. Hoang, D.R. Mikkelsen, G.V. Pereverzev, F. Ryter, O. Sauter, R.E. Waltz for very helpful discussions.

\section{References}

[1] Ward D J, Cook I, Lechon Y, Saez R, 2005 Fusion Eng. Design 75-79 1221.

[2] Greenwald M, Terry J L, Wolfe S M et al 1988 Nucl. Fusion 282199.

[3] Maisonnier D, Cook I, Sardain P et al 2005 Fusion Eng. Design 75-79 1173.

[4] Osborne T H, Leonard A W, Mahdavi M A et al 2001 Phys. Plasmas 82017.

[5] Stober J, Fuchs C, Gruber O et al 2001 Nucl. Fusion 411535.

[6] Mahdavi M A, Osborne T H, Leonard A W et al 2002 Nucl. Fusion 4252.

[7] Greenwald M 2002 Plasma Phys. Control. Fusion 44 R27.

[8] Valovic M, Rapp J, Cordey J G et al 2002 Plasma Phys. Control. Fusion 441911.

[9] Ware A A 1970 Phys. Rev. Lett. 2515.

[10] Stroth U et al 1991 Nucl. Fusion 312291.

[11] Hawryluk R J, Arunasalm V, Barnes C W et al 1991 Plasma Phys. Control. Fusion 331509.

[12] Ernst D R, Bonoli P T, Catto P J et al 2004 Phys. Plasmas 112637.

[13] Sabot R, Clairet F, Conway G D et al 2006 Plasma Phys. Control. Fusion 48 B421.

[14] Gentle K W, Richards B and Waelbroek F 1987 Plasma Phys. Control. Fusion 291077.

[15] Mertens V, Bessenrodt-Weberpals M, Dodel G et al 1990 Plasma Phys. Control. Fusion 32965.

[16] Brower D E et al 1990 Phys. Rev. Lett. 65337.

[17] Efthmion P C et al 1991 Phys. Rev. Lett. 66421.

[18] Efthmion P C et al 1991 Phys. Fluids B 32315.

[19] Wagner F and Stroth U 1993 Plasma Phys. Control. Fusion 351321.

[20] Lopes Cardozo N J 1995 Plasma Phys. Control. Fusion 37799.

[21] Hinton F L and Hazeltine R D 1976 Rev. Mod. Phys. 48239.

[22] Coppi B, Sharky N 1981 Nucl. Fusion 211363.

[23] Burrell K H, Gentle K W, Luhmann N C Jr et al 1990 Phys. Fluids B 22904.

[24] Hoang G T, Bourdelle C, Pégourié B, Schunke B et al 2003 Phys. Rev. Lett. 90155002.

[25] Zabolotsky A, Weisen H and TCV Team 2003 Plasma Phys. Control. Fusion 45735.

[26] Furno I, Weisen H and TCV Team 2003 Phys. Plasmas 102422.

[27] Weisen H, Zabolotsky A, Garbet X et al 2004 Plasma Phys. Control. Fusion 46751.

[28] Romanelli M, Hoang G T, Bourdelle C et al 2007 Plasma Phys. Control. Fusion 49935.

[29] Baker D R and Rosenbluth M 1998 Phys. Plasmas 52936.

[30] Baker D R, Wade M R, Petty C C, Rosenbluth M N et al 2000 Nucl. Fusion 401003.

[31] Weisen H, Furno I and TCV Team 2001 Nucl. Fusion 411227.

[32] Weisen H, Furno I, Alberti S et al 2002 Nucl. Fusion 42136.

[33] Peeters A G, Gruber O, Günter S et al 2002 Nucl. Fusion bf 421376.

[34] Stober J, Dux R, Gruber O et al 2003 Nucl. Fusion 431265.

[35] Angioni C, Peeters A G, Pereverzev G V et al 2003 Phys. Rev. Lett. 90205003.

[36] Angioni C, Peeters A G, Pereverzev G V et al 2003 Phys. Plasmas 103225.

[37] Garzotti L, Garbet X, Mantica P et al 2003 Nucl. Fusion 431829.

[38] Angioni C, Peeters A G, Garbet X et al 2004 Nucl. Fusion 44827.

[39] Hoang G T, Bourdelle C, Garbet X et al 2004 Phys. Rev. Lett. 93135003.

[40] Valovič N, Budny R, Garzotti L et al. 2004 Plasma Phys. Control. Fusion 461877.

[41] Weisen H, Zabolotsky A, Angioni C et al. 2005 Nucl. Fusion 45 L1.

[42] Angioni C, Peeters A G, Ryter F et al 2005 Phys. Plasmas 12040701.

[43] Peeters A G, Angioni C, Apostoliceanu M et al 2005 Nucl. Fusion 451140.

[44] Zabolotsky A, Weisen H, Karpushov A 2006 Nucl. Fusion 46594. 
[45] Zabolotsky A, Weisen H and TCV Team 2006 Plasma Phys. Control. Fusion 48369.

[46] Weisen H, Zabolotsky A, Maslov M et al 2006 Plasma Phys. Control. Fusion 47 A457.

[47] Garzotti L, Valovič M, Garbet X, Mantica P, Parail V 2006 Nucl. Fusion 46994.

[48] Fable E, Sauter O, Coda S et al 2006 Plasma Phys. Control. Fusion 481271.

[49] Valovič M, Garzotti L, Voitsekhovitch I et al 2007 Nucl. Fusion 47196.

[50] Angioni C, Carraro L, Dannert T et al 2007 Phys. Plasmas 2007 Phys. Plasmas 14055905.

[51] Angioni C, Weisen H, Kardaun O J W F et al. 2007 Nucl. Fusion 471326.

[52] Greenwald M, Angioni C, Hughes J W, Terry J and Weisen H 2007 Nucl. Fusion 47 L26.

[53] Takenaga H, Tanaka K, Muraoka K et al 2008 Nucl. Fusion 48075004.

[54] Maslov M, Angioni C and Weisen H 2009 Nucl. Fusion 49075037.

[55] Coppi B and Spight C 1978 Phys. Rev. Lett. 41551.

[56] Antonsen T, Coppi B, Englade R 1979 Nucl. Fusion 19641.

[57] Waltz R E 1986 Phys. Fluids 293684.

[58] Lee G S and Diamond P H 1986 Phys. Fluids 293291.

[59] Shaing K C 1988 Phys. Fluids 312249.

[60] Terry P W 1989 Phys. Fluids B 11932.

[61] Waltz R E and Dominguez R R 1989 Phys. Fluids B 11935.

[62] Weiland J, Jarmén A B, Nordman H 1989 Nucl. Fusion 291810.

[63] Nordman H, Weiland J, Jarmén A B 1990 Nucl. Fusion 30983.

[64] Yankov V V 1994 JETP Lett. 60171.

[65] Nycander J and Yankov V 1995 Phys. Plasmas 42874.

[66] Isichenko M B, Gruzinov A V and Diamond P H 1995 Phys. Rev. Lett. 744436.

[67] Isichenko M B, Gruzinov A V, Diamond P H and Yushmanov P N 1996 Phys. Plasmas 31916.

[68] Yankov V V and Nycander J 1997 Phys. Plasmas 42907.

[69] Naulin V, Nycander J, and Jull Rasmussen J 1998 Phys. Rev. Lett. 814148.

[70] Miskane F, Garbet X, Dezairi A, and Saifaoui D 2000 Phys. Plasmas 74197.

[71] Garbet X, Garzotti L, Mantica P et al 2003 Phys. Rev. Lett. 91035001.

[72] Baker D R 2004 Phys. Plasmas 11992.

[73] Garbet X, Dubuit N, Asp E et al 2005 Phys. Plasmas 12082511.

[74] Waltz R E, Dominguez R R, and Hammett G W 1992 Phys. Fluids B 43138.

[75] Waltz R E, Staebler G M, Dorland W et al 1997 Phys. Plasmas 42482.

[76] Kotschenreuther M, Rewoldt G, and Tang W M 1995 Comput. Phys. Commun. 88128

[77] Dorland W, Jenko F, Kotschenreuther M, Rogers B N 2000 Phys. Rev. Lett. 855579

[78] Jenko F, Dorland W, Kotschenreuther M and Rogers B N 2000 Phys. Plasmas 71904.

[79] Dannert T and Jenko F 2005 Phys. Plasmas 12072309.

[80] Bourdelle C, Garbet X, Hoang G T, Ongena J, and Budny R V 2002 Nucl. Fusion 42892.

[81] Candy J and Waltz R E 2003 J. Comput. Phys. 186545.

[82] Candy J and Waltz R E 2003 Phys. Rev. Lett. 91045001.

[83] Bourdelle C 2005 Plasma Phys. Control. Fusion 47 A317.

[84] Jenko F, Dannert T, and Angioni C 2005 Plasma Phys. Control. Fusion 47 B195.

[85] Estrada-Mila C, Candy J, Waltz R E 2005 Phys Plasmas 12 022305. Erratum 2005 Phys. Plasmas 12049902.

[86] Angioni C, Peeters A G, Jenko F, and Dannert T 2005 Phys. Plasmas 12112310 (2005).

[87] Hallatschek K, Dorland W 2005 Phys. Rev. Lett. 95055002.

[88] Estrada-Mila C, Candy J, Waltz R E 2006 Phys Plasmas 13074505.

[89] Bourdelle C, Garbet X, Imbeaux F et al 2007 Phys. Plasmas 14112501.

[90] Fable E, Angioni C and Sauter O 2008 Plasma Phys. Control. Fusion 50115005.

[91] Angioni C, Candy J, Fable E, Maslov M, Waltz R E, Weisen H 2009 Phys. Plasmas 16, 060702.

[92] Terry P W and Gatto R 2006 Phys. Plasmas 13062309.

[93] Angioni C and Sauter O 2000 Phys, Plasmas 71224.

[94] Coppi B and Pegoraro F 1977 Nucl. Fusion 175. 
[95] Romanelli F 1989 Phys. Fluids B 11018.

[96] Coppi B, Rewoldt G 1974 Phys. Rev. Lett. 331329.

[97] Antonsen T and Lane B 1980 Phys. Fluids 231205.

[98] Frieman E A and Chen L 1982 Phys. Fluids 25502.

[99] Hahm T S 1988 Phys. Fluids 312670.

[100] Scott B D 2007 Plasma Phys. Control. Fusion 49 S25.

[101] Fable E, Angioni C and Sauter O 2009 Plasma Phys. Control. Fusion submitted.

[102] Connor J W, Hastie R J and Taylor J B 1978 Phys. Rev. Lett. 40396.

[103] Casati A, Bourdelle C, Garbet X Imbeaux F et al 2009 Nucl. Fusion 49085012.

[104] Dimits A M, Bateman G, Beer M A et al 2000 Phys. Plasmas 7969.

[105] Ernst D. R. et al 2007 21st IAEA FEC, Chengdu, China, 2006, IAEA, Vienna, 2007, IAEA-CN149/TH/1-3; http://www-pub.iaea.org/MTCD/Meetings/FEC2006/th_1-3.pdf.

[106] Braginskii S I 1966 in Reviews of Plasma Physics, edited by Leontovich M A (Consultant Bureau, New York 1966) Vol. 1205.

[107] Jenko F 2000 Phys. Plasmas 7514.

[108] Staebler G M, Kinsey J and Waltz R E 2007 Phys. Plasmas 14055909.

[109] Angioni C and Peeters A G 2006 Phys. Rev. Lett. 96095003.

[110] Eriksson A and Weiland J 2005 Phys. Plasmas 12092509.

[111] Candy J 2005 Phys. Plasmas 12072307.

[112] Angioni C, Dux R, Fable F et al 2007 Plasma Phys. Control. Fusion 492027.

[113] Waltz R E, Casati A and Staebler G M, 2009 Phys. Plasmas 16072303.

[114] Greenwald M et al 2007 Fusion Sci. Technol. 51266.

[115] Zastrow K -D et al 2004 Plasma Phys. Control. Fusion 46 B255.

[116] Gilgenbach R M et al 1980 Phys. Rev. Lett. 44647.

[117] Riviere A C 1986 Nucl. Fusion 281995.

[118] TFR Group 1988 Nucl. Fusion 281995.

[119] Razumova K A et al 2008 Plasma Phys. Control. Fusion 50105004.

[120] Razumova K A, Andreev V F, Kislov A Ya et al 2009 Nucl. Fusion 49065011.

[121] Maslov M, Weisen H et al EPS 2006.

[122] Porte L, Coda S, Alberti S et al 2007 Nucl. Fusion 47952.

[123] Conway G D 2008 Plasma Phys. Control. Fusion 50124026.

[124] Pereverzev G V, Angioni C, Peeters A G and Zolotukhin O V 2005 Nucl. Fusion 45221.

[125] Weisen $\mathrm{H}$ et al 2006 21st IAEA FEC, Chengdu, China 16-20.10.2006. 